\title{
Radiative forcing of anthropogenic aerosols on cirrus clouds using a hy- brid ice nucleation scheme
}

\author{
Jialei Zhu and Joyce E. Penner \\ Correspondence to: Jialei Zhu (zhujialei@tju.edu.cn)
}

The copyright of individual parts of the supplement might differ from the CC BY 4.0 License. 


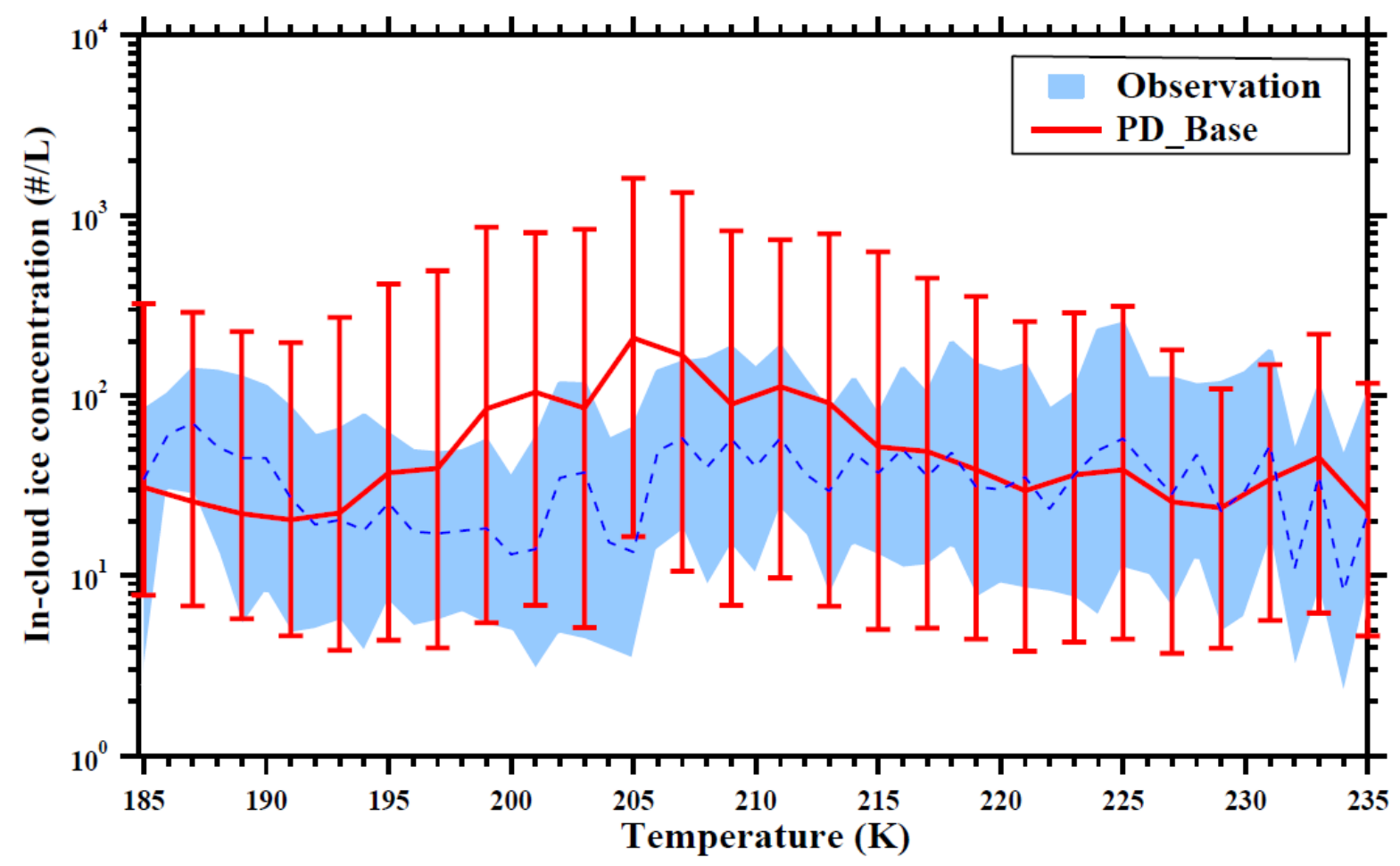

Figure S1. The median of in-cloud ice crystal number concentration (red line) versus temperature from PD_Base case with error bars of 25th and 75th percentiles. The background shaded region shows the 25th-75th percentiles with the median value (blue dashed line) from a set of observations compiled by Krämer et al. $(2009,2020)$ 
(a)

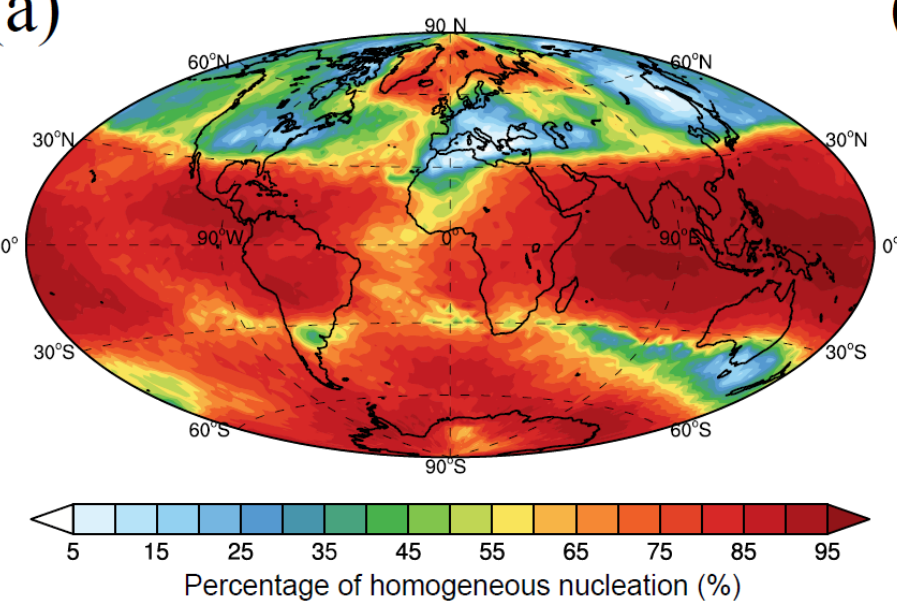

(c)

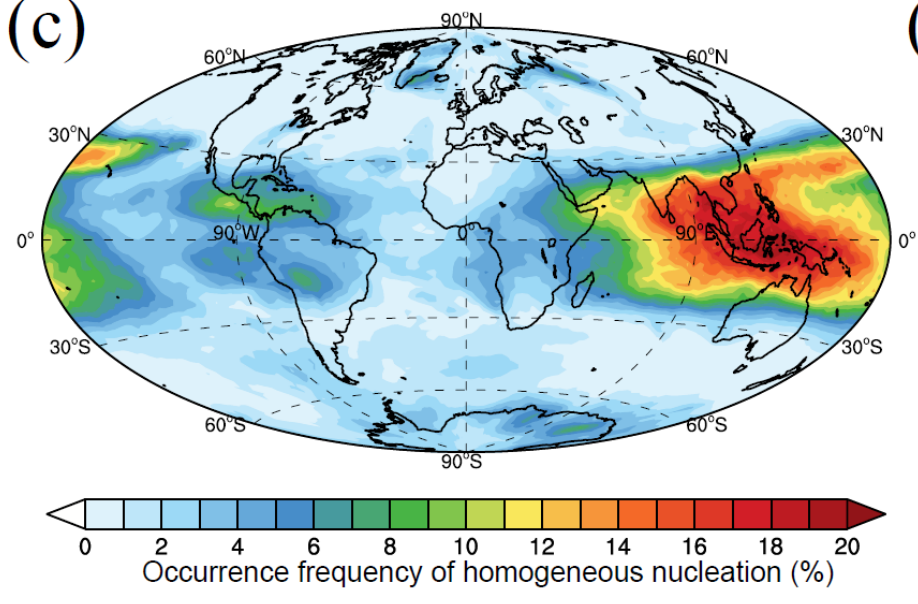

(b)

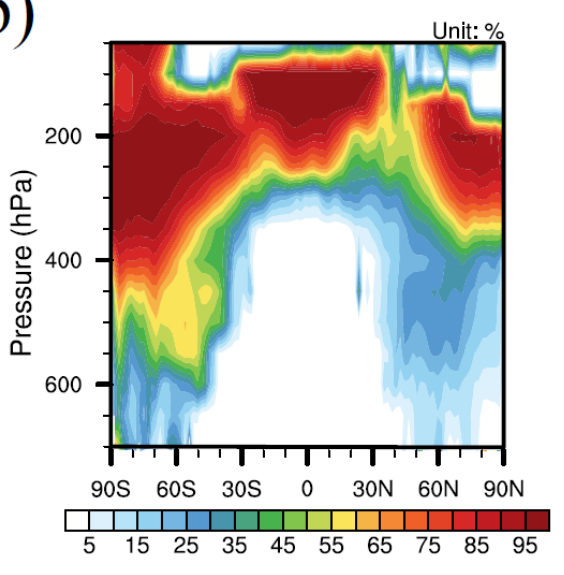

(d)

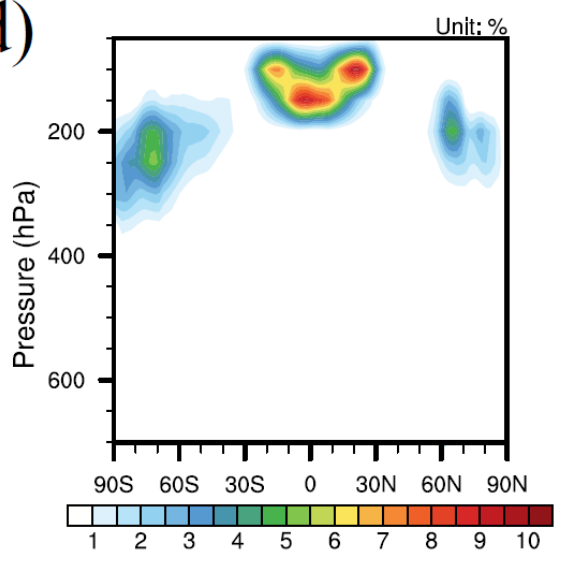

Figure S2. Vertically integrated percentage of new ice formed from homogeneous nucleation (a) and the cloud-weighed occurrence frequency of homogeneous nucleation (c) in the PD_Base case. The zonal average plots of percentage of new ice formed from homogeneous nucleation (b) and cloud-weighed occurrence frequency of homogeneous nucleation (d) in the PD_Base case. 
(a)

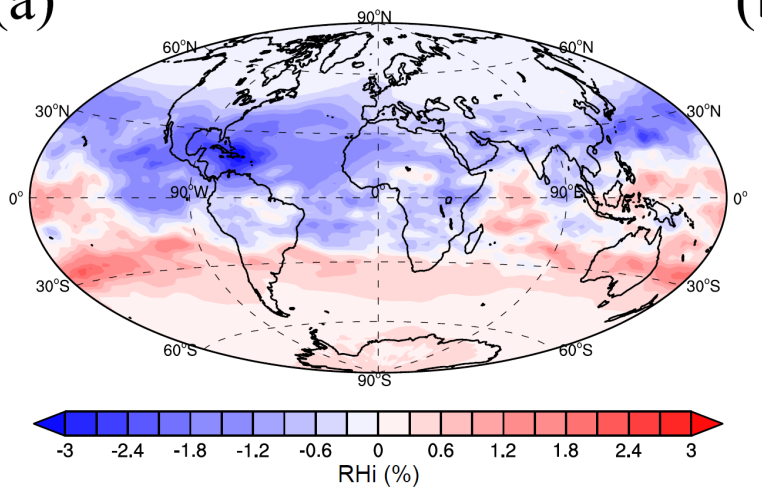

(b)

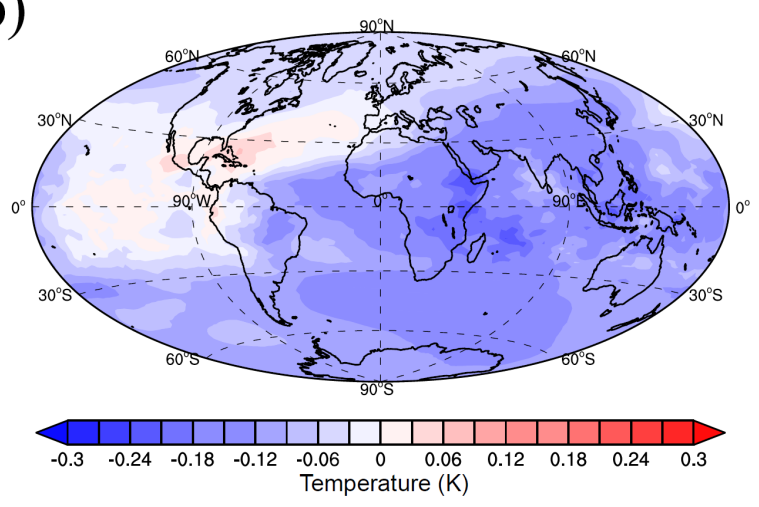

Figure S3. The annual average change in the RHi (a) and temperature (b) at 150 hPa for the difference between the PD_Base and PI_cSoot cases. 
(a)

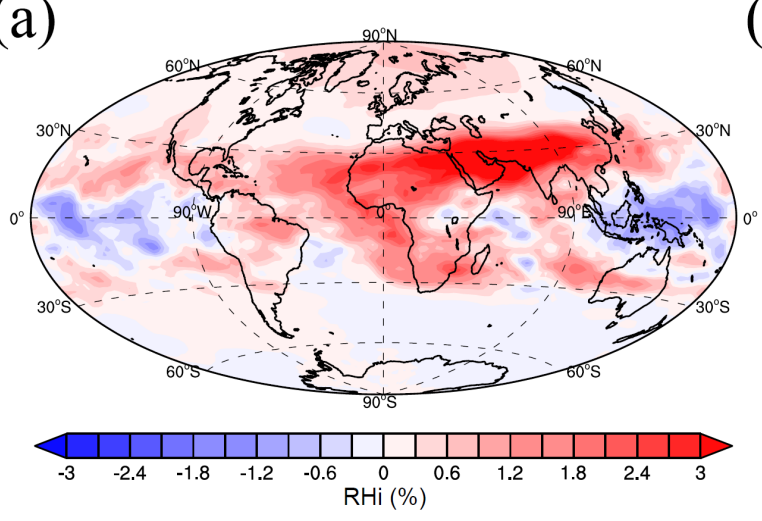

(c)

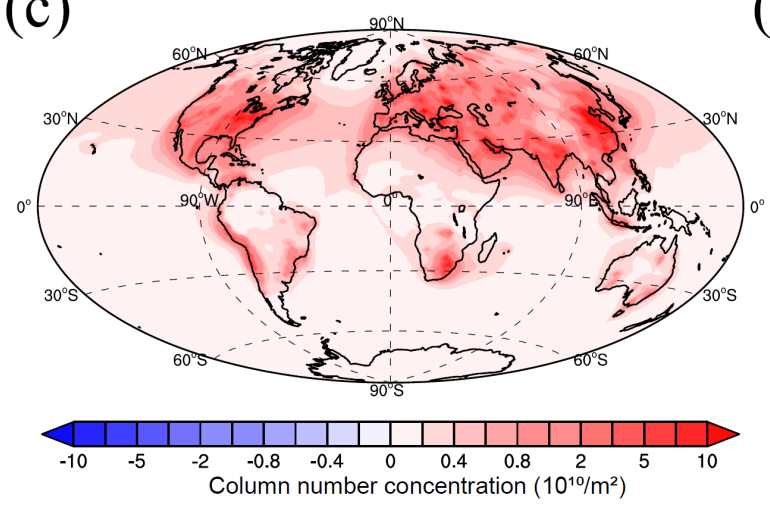

(b)

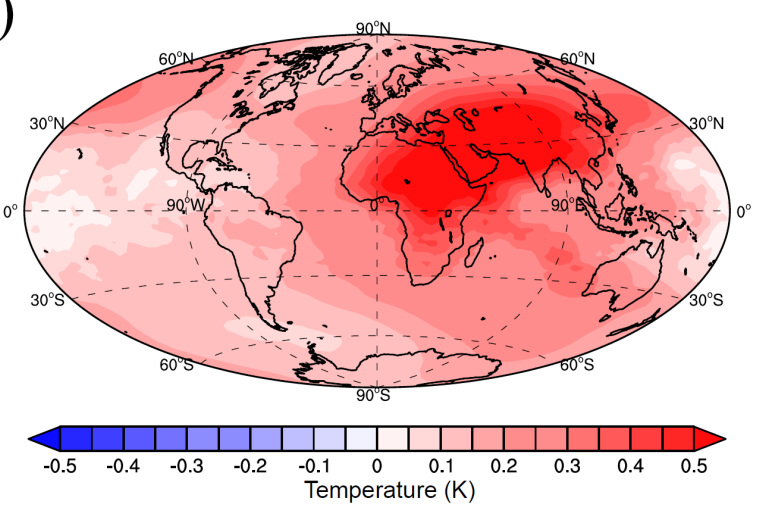

(d)

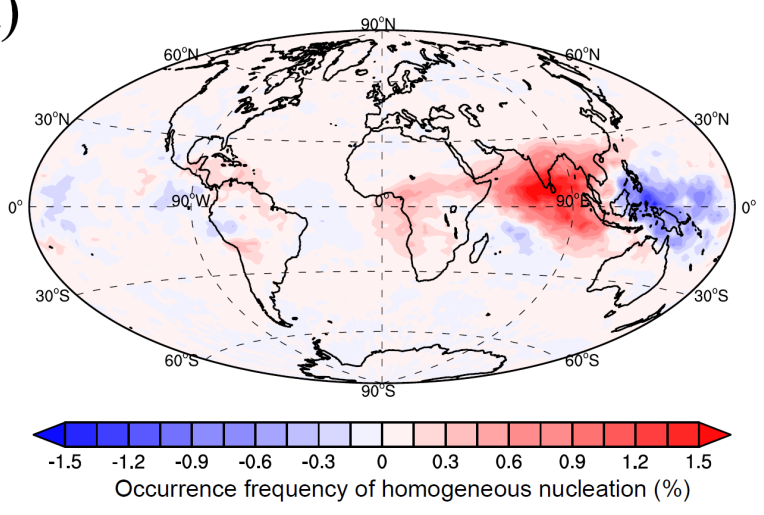

Figure S4. The annual average change in the RHi (a), temperature (b) and occurrence frequency of homogeneous nucleation (d) at $150 \mathrm{hPa}$ as well as vertically integrated number concentration of sulfate in the Aitken and accumulation mode (c) for the difference between the PD_Base and PI_SO4 cases. 


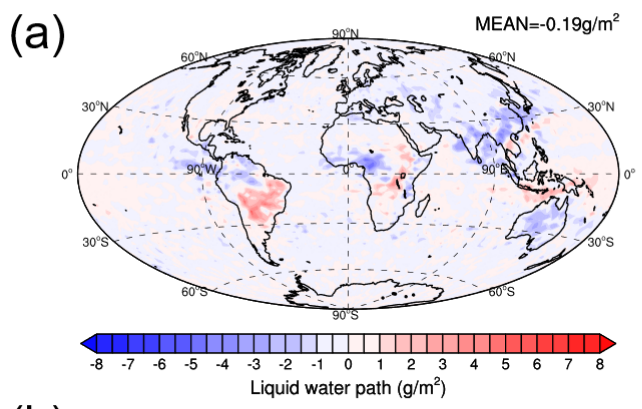

(b)

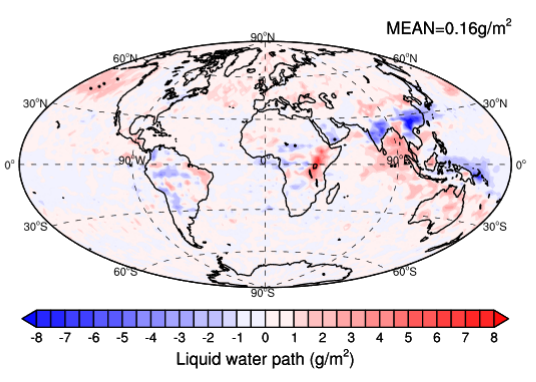

(c)

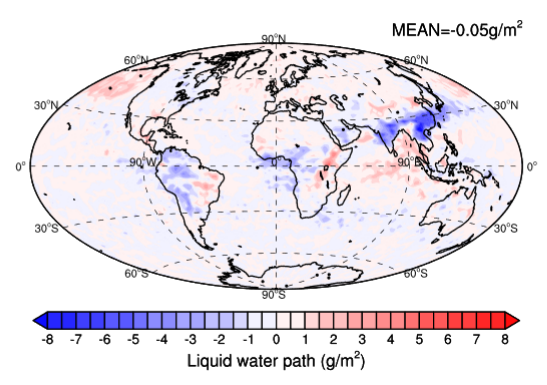

Figure S5. Annual mean plots of the change in vertically integrated averaged i liquid water path for the difference between the PD_Base and PI_cSoot cases (a,), PD_Base and PI_SO4 cases (b) as well as the PD_Base and PI_ALL cases (c). Differences significant at the $90 \%$ level according to a Student's $t$ test are depicted by points. 
(a)

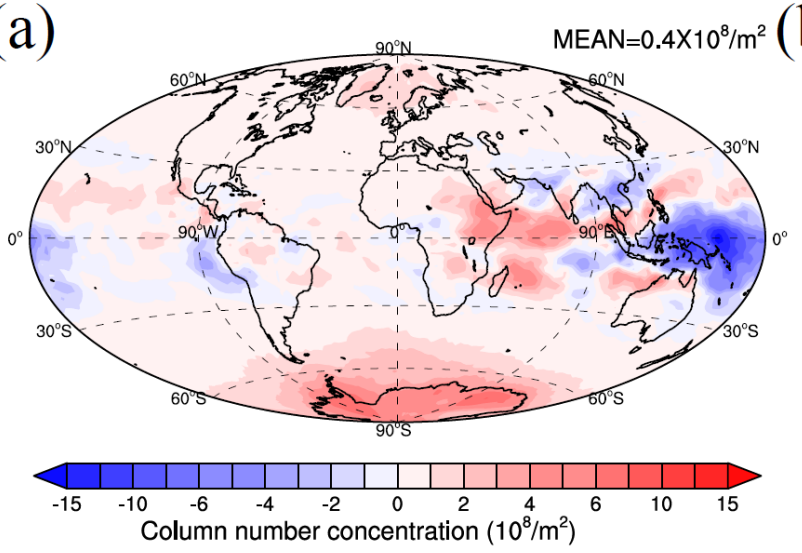

(c)

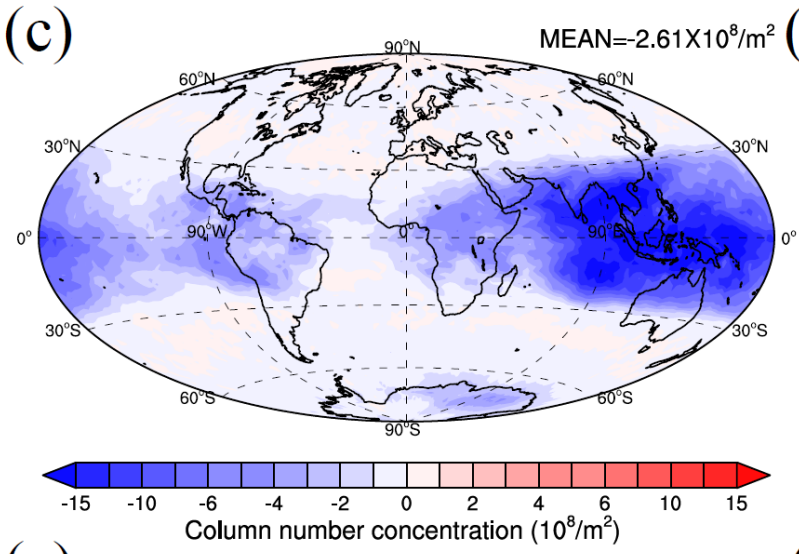

(e)

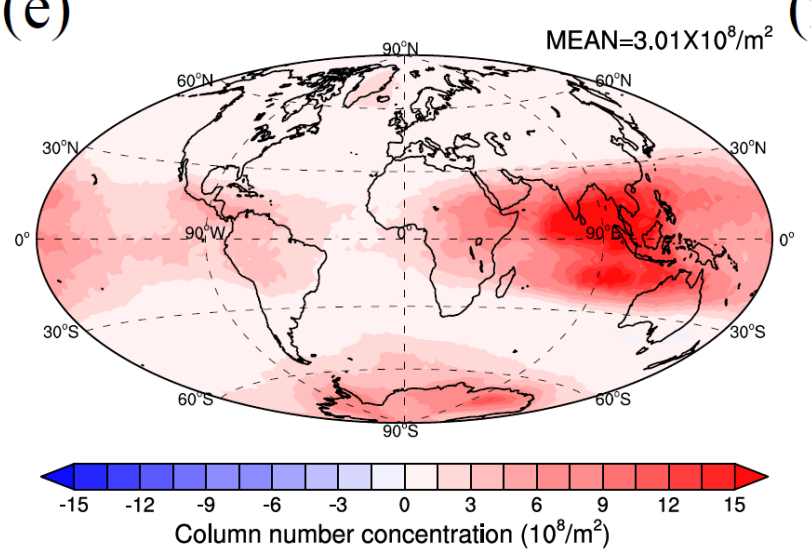

(b)

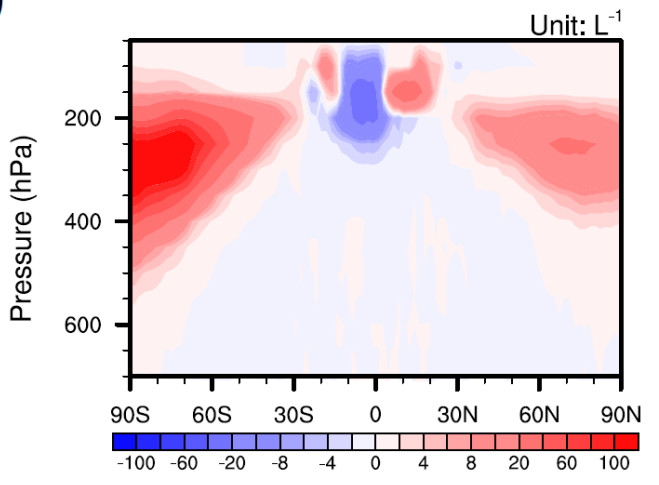

(d)

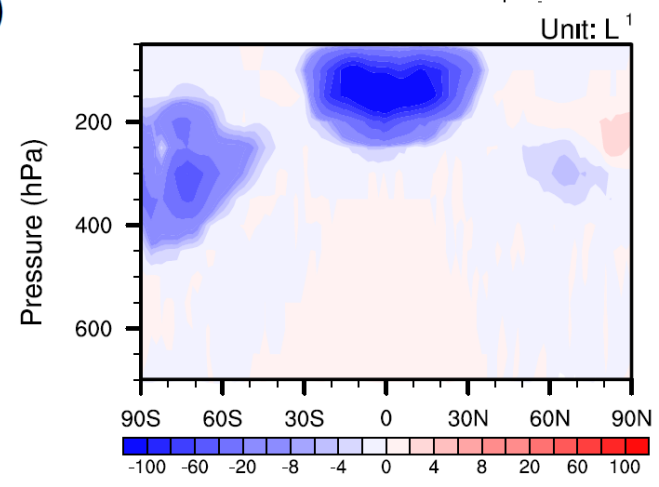

(f)

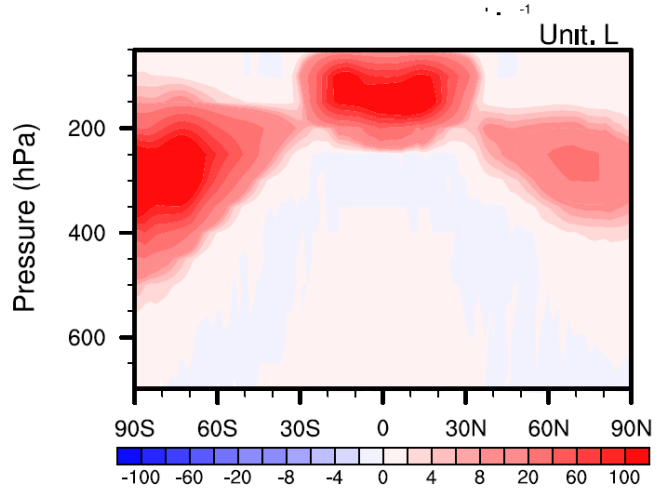

Figure S6. The annual average change in column number concentration (a, c, e) and zonal average number concentration ( $b, d, f)$ of total ice $(a, b)$, ice from homogeneous nucleation (c, d) and ice from heterogeneous nucleation (e, f) for the difference between the PD_SOA and PD_Base cases. Differences significant at the $90 \%$ level of according to a Student's $t$ test are depicted by points. 
(a)

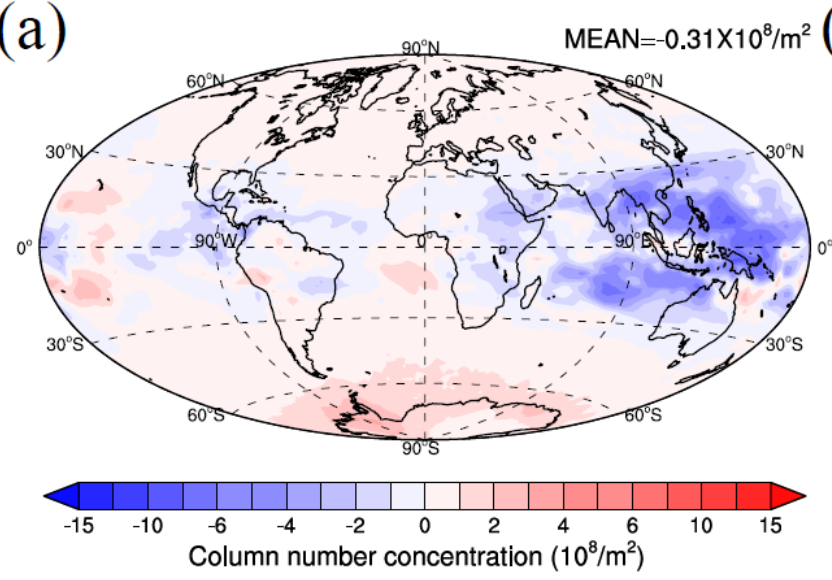

(c)

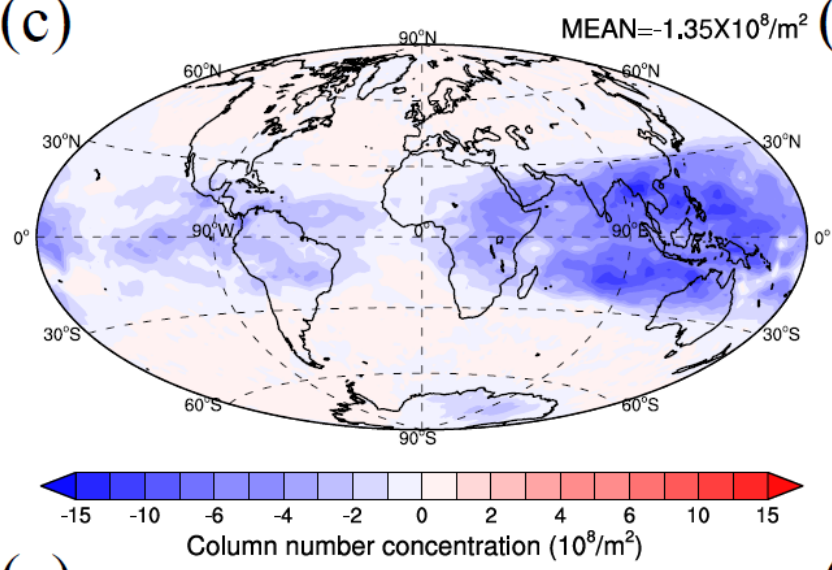

(e)

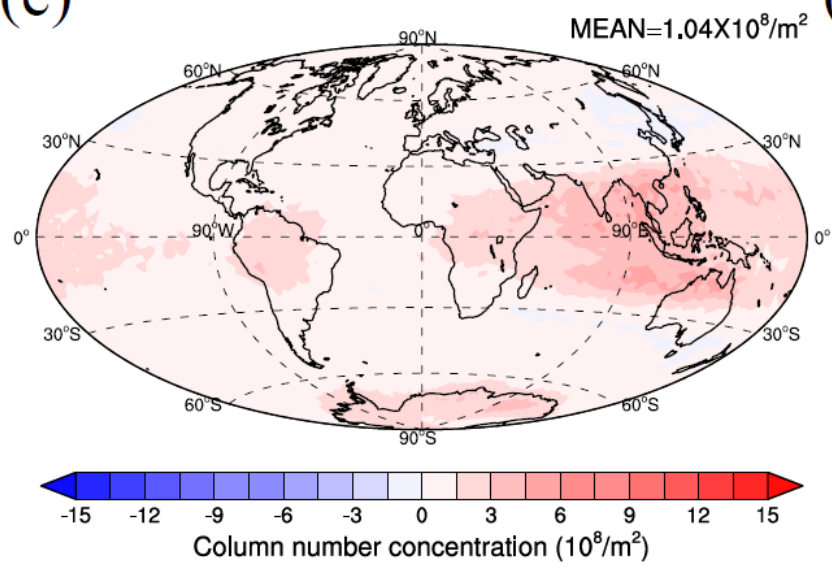

(b)

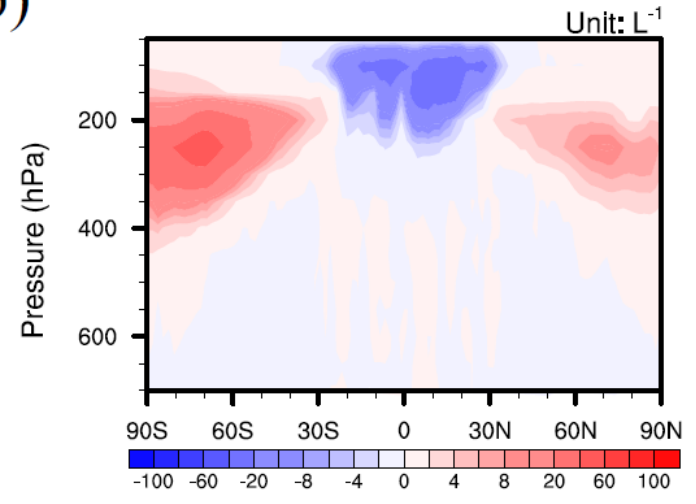

(d)

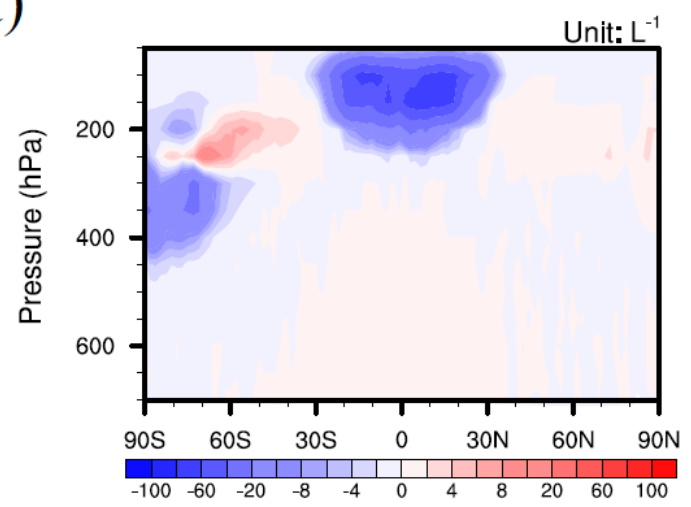

(f)

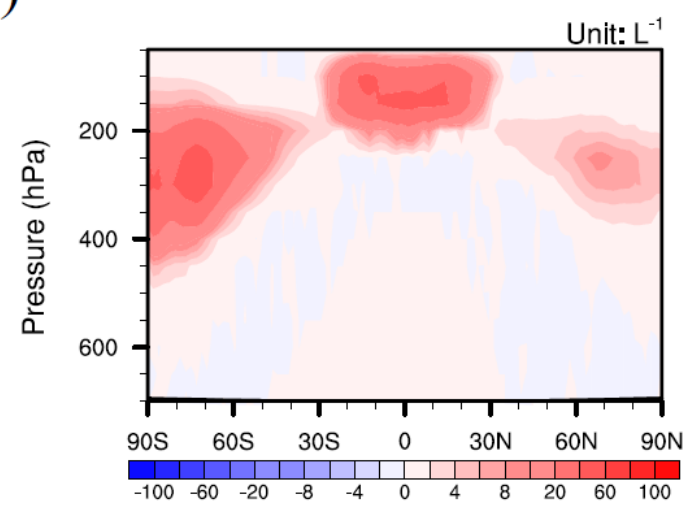

Figure S7. As in Figure S6 but for the difference between the PI_SOA and PI_ALL cases. Differences significant at the $90 \%$ level according to a Student's $t$ test are depicted by points. 


\section{Text S1 Sensitivity experiments with high concentration of INPs from dust}

In order to examine the influence of heterogeneous nucleation on radiative forcing, sensitivity experiments with a high concentration of INPs from dust were conducted. In these experiments $100 \%$ of dust was assumed to act as an INP with all anthropogenic emissions for the present day (PD_HDust) and preindustrial periods (PI_HDust). In comparison, only dust with fewer than 3 monolayers of sulfate coating were assumed to act as INPs in PD_Base and PI_ALL cases. The global average $N i$ from heterogeneous nucleation is increased by about a factor of $\sim 4$ due to the high concentration of INPs from dust in PD_HDust case compared to PD_Base and there is a remarkable increase of $\mathrm{Ni}$ from heterogeneous nucleation in the main regions with dust sources like Sahara and Taklimakan as well as downwind from these regions (Figure S8b). In addition, homogeneous nucleation is inhibited by the increase in the INPs. The global average $N i$ from homogeneous nucleation is decreased by $27 \%$ in PD_HDust compared to PD_Base (Figure S8a). As a result, the assumption of $100 \%$ of dust acting as INPs increases $N i$ in the mid to high latitudes of the $\mathrm{NH}\left(30^{\circ} \mathrm{N} \sim 90^{\circ} \mathrm{N}\right)$ where heterogeneous nucleation dominates, while $\mathrm{Ni}$ over the tropics is decreased due to the inhibition of homogeneous nucleation (Figure $\mathrm{S} 9 \mathrm{a})$. In total the global average $N i$ in the PD_HDust case is $11 \%$ lower that in the PD_Base case. The increased INPs from dust have a similar influence on $N i$ in the PI atmosphere, which leads to $12 \%$ lower global average $N i$ in the PI_HDust case than that in the PI_ALL case (Figure S9b). In this sensitivity experiment, $\mathrm{Ni}$ increases significantly in south Asia and the north Indian Ocean from the PI to PD atmosphere (Figure S10a, PD_HDust-PI_HDust), which is somewhat smaller than the increase in the base experiment (PD_Base-PI_ALL) due to the decrease in homogeneous nucleation. The decrease in $\mathrm{Ni}$ off the coast of east Asia from PI to $\mathrm{PD}$ in this sensitivity experiment is not as significant as that in base experiment (compare Figure S10a and Figure 5a) because the high concentration of INPs from 
dust in the PI atmosphere leads to a smaller influence on $N i$ from anthropogenic emissions compared to the base experiment. As a result, the global average increase in $\mathrm{Ni}$ is somewhat larger from PI_HDust to PD_HDust $\left(0.57 \times 10^{8} \mathrm{~m}^{-2}\right.$, Figure S10a) than the increase in base experiment $\left(0.49 \times 10^{8} \mathrm{~m}^{-2}\right.$, Figure $\left.5 \mathrm{a}\right)$.

The more positive change in $N i$ from PI to PD in this sensitivity experiment leads to a less negative change in the IWP than that in base experiment (compare Figure $\mathrm{S} 10 \mathrm{~b}$ and Figure 6e). Due to the larger change in $N i$ in this sensitivity experiment than that in the base experiment, the SRF is less positive (Figure S10c vs Figure 9a) and the LRF is less negative (Figure S10d vs Figure 9b) in the mid to high latitudes of the NH. SRF and LRF are smaller in south Asia and the north Indian Ocean in the sensitivity experiment compared to the base experiment. As a result, the NRF between PD_HDust and PI_HDust is $-0.16 \pm 0.06 \mathrm{~W} \mathrm{~m}^{-2}$ (Figure S10e), which is less negative than the NRF in the base case $\left(-0.20 \pm 0.05 \mathrm{~W} \mathrm{~m}^{-2}\right.$, Figure 9e). The increase in INPs from dust leads to a less negative NRF in the mid to high latitudes of the NH $\left(30^{\circ} \mathrm{N} \sim 90^{\circ} \mathrm{N}\right)$ and a less positive NRF between $20^{\circ} \mathrm{N} \sim 30^{\circ} \mathrm{N}$ (Figure S10f). 
(a)

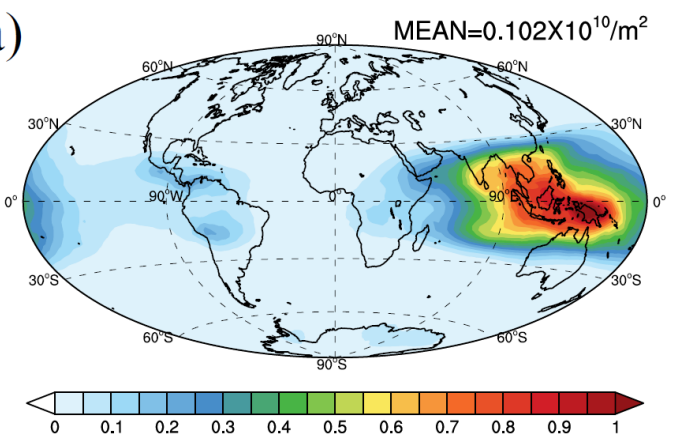

(b)

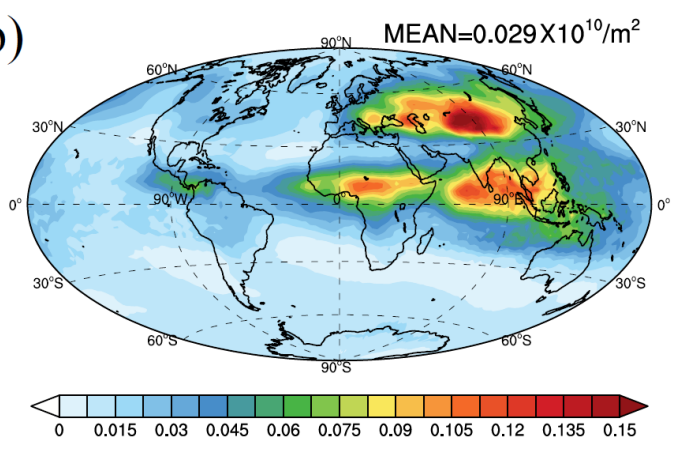

Figure S8. The vertically integrated $N i$ from homogeneous nucleation (a) and from heterogeneous nucleation (b) for the PD_HDust case.

(a) PD

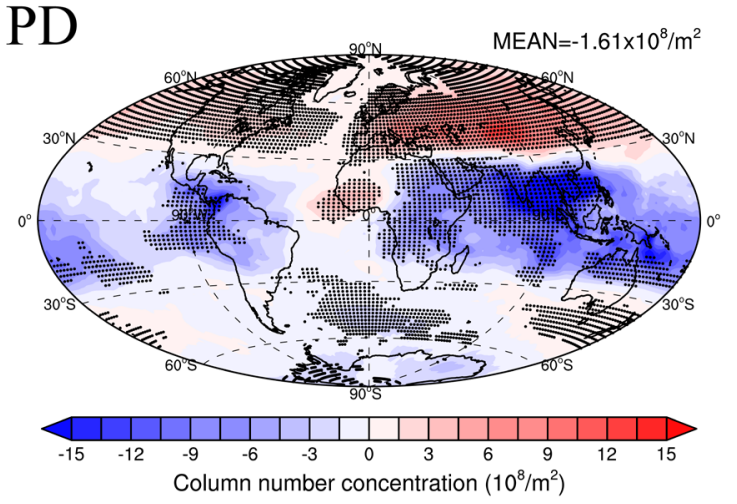

(b) PI

MEAN $=-1.68 \times 10^{8} / \mathrm{m}^{2}$

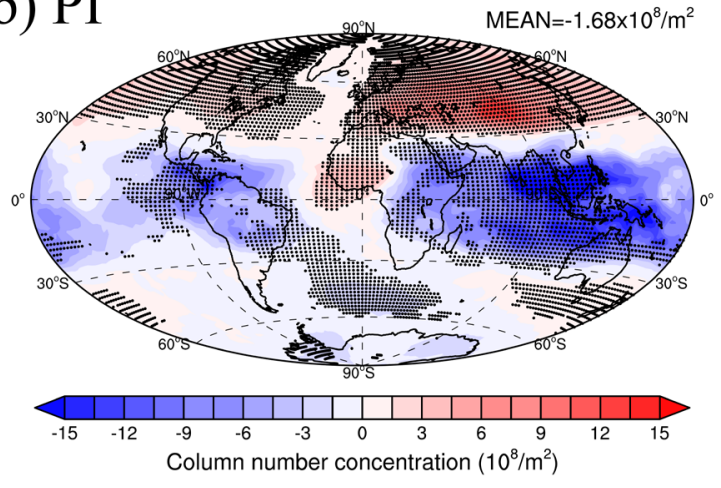

Figure S9. The difference in the column number concentration of Ni between PD_HDust and PD_Base (a) as well as PI_HDust and PI_ALL (b). Differences significant at the $90 \%$ level according to a Student's t test are depicted by points. 
(a)

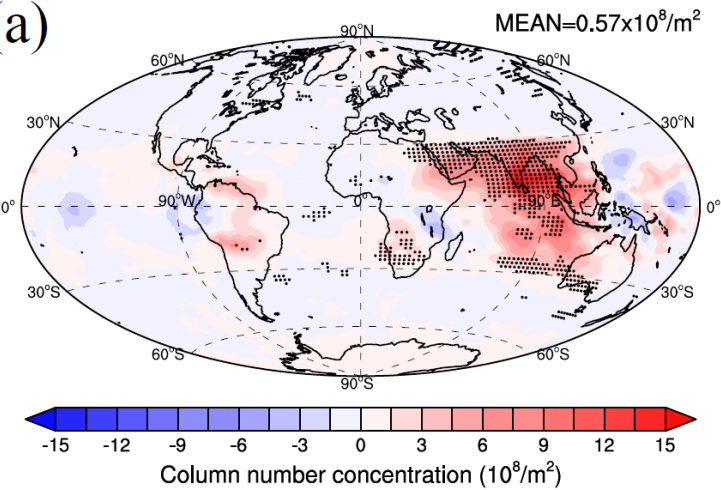

(c)

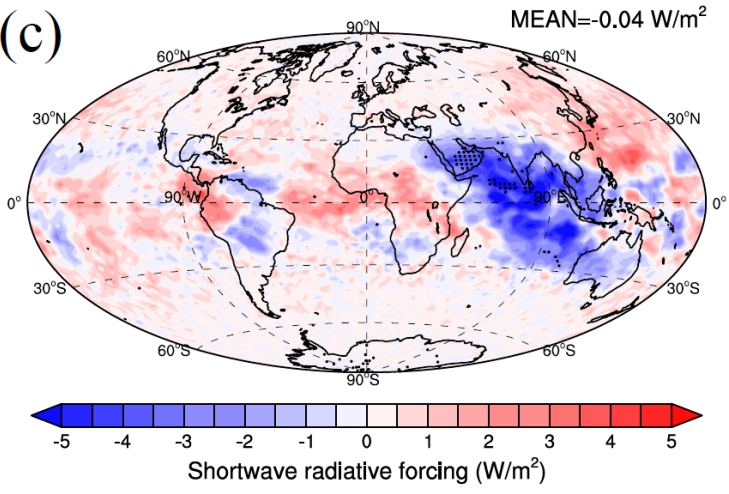

(e)

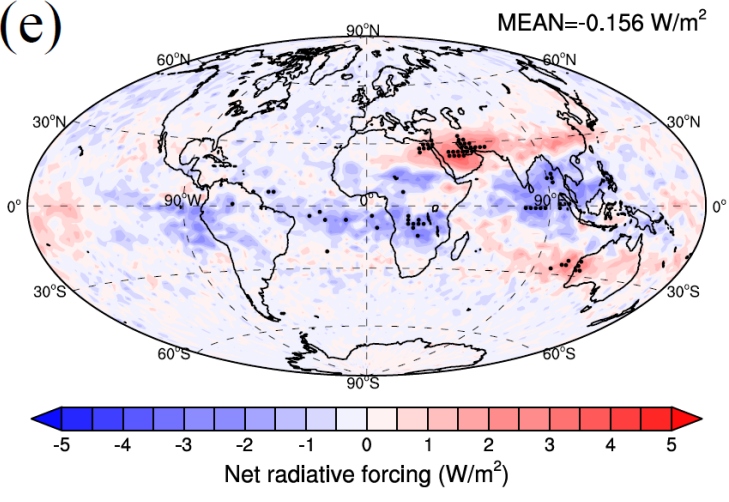

(b)

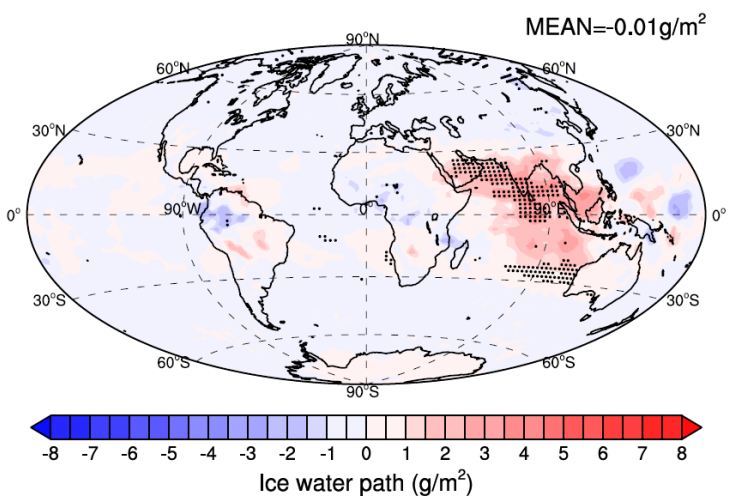

(d)

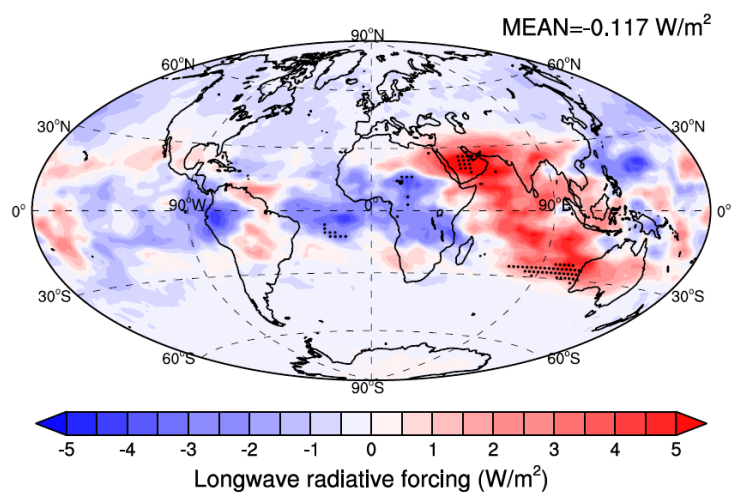

(f)

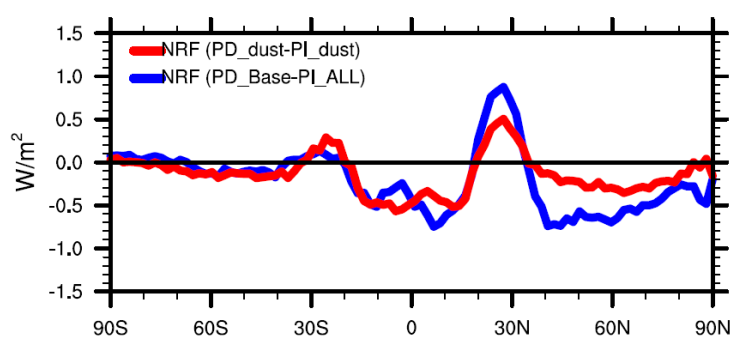

Figure S10. Annual mean plots of the changes in vertical integrated Ni (a), ice water path (b), shortwave radiative forcing (c), longwave radiative forcing (d), and all-sky net radiative forcing (e) as well as longwave radiative forcing (f, LRF, green dashed line), shortwave radiative forcing (f, SRF, blue dotted line) and allsky net radiative forcing (f, NRF, red solid line) versus latitude for the difference between the PD_HDust and PI_HDust cases. Differences significant at the 90\% level according to a Student's $t$ test are depicted by points. 